\title{
Iterative Algorithms for the Split Problem and Its Convergence Analysis
}

\author{
Zhangsong Yao, ${ }^{1}$ Arif Rafiq, ${ }^{2}$ Shin Min Kang, ${ }^{3}$ and Li-Jun Zhu ${ }^{4}$ \\ ${ }^{1}$ School of Mathematics \& Information Technology, Nanjing Xiaozhuang University, Nanjing 211171, China \\ ${ }^{2}$ Department of Mathematics, Lahore Leads University, Lahore 54810, Pakistan \\ ${ }^{3}$ Department of Mathematics and RINS, Gyeongsang National University, Jinju 660-701, Republic of Korea \\ ${ }^{4}$ School of Mathematics and Information Science, Beifang University of Nationalities, Yinchuan 750021, China
}

Correspondence should be addressed to Shin Min Kang; smkang@gnu.ac.kr

Received 12 May 2014; Accepted 18 May 2014; Published 28 May 2014

Academic Editor: Jen-Chih Yao

Copyright (C) 2014 Zhangsong Yao et al. This is an open access article distributed under the Creative Commons Attribution License, which permits unrestricted use, distribution, and reproduction in any medium, provided the original work is properly cited.

Now, it is known that the split common fixed point problem is a generalization of the split feasibility problem and of the convex feasibility problem. In this paper, the split common fixed point problem associated with the pseudocontractions is studied. An iterative algorithm has been presented for solving the split common fixed point problem. Strong convergence result is obtained.

\section{Introduction}

Now, we know that the convex feasibility problem can be formulated as finding a point $x^{\dagger}$ such that

$$
x^{\dagger} \in \bigcap_{i=1}^{n} \mathbb{C}_{i}, \quad n \in \mathbb{N},
$$

where $\mathbb{C}_{i}(\neq \emptyset)$ is a closed convex subset of a Hilbert space $\mathbb{T}$. The convex feasibility problem has extensive applications in many applied disciplines such as signal processing, biomedical engineering, and communications. For related works, please see [1-3].

If $n=2$ in (1), then a special case of (1) is the following split feasibility problem.

Problem 1. The split feasibility problem: let $\mathbb{U}_{1}$ and $\mathbb{H}_{2}$ be two Hilbert spaces. Let $\mathbb{C}_{1} \subset \mathbb{Q}_{1}$ and $\mathbb{C}_{2} \subset \mathbb{Q}_{2}$ be two nonempty closed convex sets. Let $A: \mathbb{U}_{1} \rightarrow \mathbb{\square}_{2}$ be a bounded linear operator. The split feasibility problem is

$$
\text { find a vector } x^{\dagger} \in \mathbb{C}_{1} \text { such that } A x^{\dagger} \in \mathbb{C}_{2} \text {. }
$$

Such problem arises in the intensity-modulated radiation therapy. In the finite-dimensional space, Censor and Elfving
[4] firstly constructed the following iterative algorithm to solve (2):

$$
x_{m+1}=A^{-1} \operatorname{proj}_{\mathbb{C}_{2}}\left(\operatorname{proj}_{A\left(\mathbb{C}_{1}\right)}\left(A x_{m}\right)\right), \quad m \in \mathbb{N},
$$

where $\mathbb{C}_{1} \subset \mathbb{R}^{n}$ and $\mathbb{C}_{2} \subset \mathbb{R}^{n}$ are closed convex sets and $A$ is an $n \times n$ matrix.

However, we note that calculating inverse $A^{-1}$ is very time-consuming, if the dimension $n$ is large. For overcoming this problem, Byrne [5] introduced the following more popular algorithm:

$$
x_{m+1}=\operatorname{proj}_{\mathbb{C}_{1}}\left(x_{m}-\Phi A^{T}\left(I-\operatorname{proj}_{\mathbb{C}_{2}}\right) A x_{m}\right), \quad m \in \mathbb{N},
$$

where $A^{T}$ denotes the transposition of $A$. Consequently, (4) and its variant have been studied extensively. For related results, please refer to [6-13].

In the case where $\mathbb{C}_{1}$ and $\mathbb{C}_{2}$ in (2) are the fixed point sets of nonlinear operators, problem (2) is called by Censor and Segal [14] the split common fixed point problem.

Problem 2. The split common fixed point problem: this problem is to find a fixed point $x^{\dagger}$ of an operator $S$ in the space $\mathbb{Q}_{1}$ such that its image $A x^{\dagger}$ under a linear transformation $A$ 
is a fixed point $y^{\dagger}$ of another operator $T$ in the image space $\mathbb{H}_{2}$. Namely, find a vector $x^{\dagger} \in H$ such that

$$
x^{\dagger} \in \operatorname{Fix}(S), \quad y^{\dagger}=A x^{\dagger} \in \operatorname{Fix}(T),
$$

where $\operatorname{Fix}(S)$ and $\operatorname{Fix}(T)$ denote the fixed point sets of

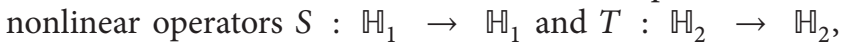
respectively.

A natural idea is to apply (4) to the split common fixed point problem (5). That is, taking $\mathbb{C}_{1}=\operatorname{Fix}(S)$ and $\mathbb{C}_{2}=$ $\operatorname{Fix}(T)$ in (4), we get

$$
\begin{aligned}
x_{m+1}=\operatorname{proj}_{\mathrm{Fix}(S)} & \left(x_{m}-\varpi A^{T}\right. \\
& \left.\times\left(I-\operatorname{proj}_{\mathrm{Fix}(T)}\right) A x_{m}\right), \quad m \in \mathbb{N} .
\end{aligned}
$$

However, $\operatorname{proj}_{\mathrm{Fix}(S)}$ and $\operatorname{proj}_{\mathrm{Fix}(T)}$ are generally not easy to calculate. Thus, (6) may fail. We have to find new algorithm to solve (5). In this respect, Censor and Segal [14] proposed the following iterative method: for any initial guess $x_{1} \in \mathbb{Z}_{1}$, define a sequence $\left\{x_{m}\right\}$ by

$$
x_{m+1}=S\left(x_{m}-\lambda A^{*}(I-T) A x_{m}\right), \quad m \in \mathbb{N},
$$

where $S$ and $T$ are directed operators. Moudafi [15] relaxed (7) to the following form:

$$
\begin{gathered}
y_{m}=x_{m}-\Phi A^{*}(I-T) A x_{m}, \\
x_{m+1}=\left(1-\alpha_{m}\right) y_{m}+\alpha_{m} S\left(y_{m}\right), \quad m \in \mathbb{N},
\end{gathered}
$$

where $U$ and $T$ are demicontractive operators.

Note that (7) and (8) have weak convergence. Some strong convergence results have been given in the literature; see, for instance, $[16,17]$. In the present paper, we consider an interesting respect: could we extend the classes of directed and demicontractive operators to the class of pseudocontractive mappings?

Our main purpose of this paper is to solve the above problem. We construct an iterative algorithm in which the involved operators are pseudocontractions and show its strong convergence.

\section{Definitions and Lemmas}

In this section, we collect some definitions and lemmas. Let $\mathbb{W}$ be a real Hilbert space with inner product $\langle\cdot, \cdot\rangle$ and norm $\|\cdot\|$, respectively. Let $(\mathbb{U} \supset) \mathbb{E} \neq \emptyset$ be a closed convex set.

Definition 3. An operator $T: \mathbb{E} \rightarrow \mathbb{E}$ is called Lipschitzian, if

$$
\left\|T x^{\ddagger}-T y^{\ddagger}\right\| \leq L\left\|x^{\ddagger}-y^{\ddagger}\right\|,
$$

for some $L>0$ and all $x^{\ddagger}, y^{\ddagger} \in \mathbb{E}$.

In this case, we call TL-Lipschitzian continuous. If $L=1$ in (9), we call $T$ nonexpansive.
Definition 4. An operator $T: \mathbb{E} \rightarrow \mathbb{E}$ is called a directed operator, if

$$
\left\langle T x^{\dagger}-x^{\dagger}, T x^{\dagger}-x\right\rangle \leq 0
$$

equivalently,

$$
\left\|T x^{\dagger}-x\right\|^{2} \leq\left\|x^{\dagger}-x\right\|^{2}-\left\|T x^{\dagger}-x^{\dagger}\right\|^{2}
$$

for all $x^{\dagger} \in \mathbb{E}$ and $x \in \operatorname{Fix}(T)$, the fixed points set of $T$.

Definition 5. An operator $T: \mathbb{E} \rightarrow \mathbb{E}$ is called a demicontractive operator, if

$$
\begin{array}{r}
\left\|T x^{\dagger}-x\right\|^{2} \leq\left\|x^{\dagger}-x\right\|^{2}+\kappa\left\|T x^{\dagger}-x^{\dagger}\right\|^{2}, \\
\forall x^{\dagger} \in \mathbb{E}, x \in \operatorname{Fix}(T),
\end{array}
$$

where $\kappa \in(0,1)$

From the above definitions, we note that the class of demicontractive operators contains important operators such as the directed operators and the nonexpansive operators with fixed points. Such a class of operators is fundamental because they include many types of nonlinear operators arising in applied mathematics and optimization; see, for example, [18] and references therein.

Definition 6. An operator $T: \mathbb{E} \rightarrow \mathbb{E}$ is called pseudocontractive, if

$$
\left\langle T x^{\dagger}-T y^{\dagger}, x^{\dagger}-y^{\dagger}\right\rangle \leq\left\|x^{\dagger}-y^{\dagger}\right\|^{2}
$$

equivalently,

$$
\left\|T x^{\dagger}-T y^{\dagger}\right\|^{2} \leq\left\|x^{\dagger}-y^{\dagger}\right\|^{2}+\left\|(I-T) x^{\dagger}-(I-T) y^{\dagger}\right\|^{2}
$$

for all $x^{\dagger}, y^{\dagger} \in \mathbb{E}$.

It is obvious that the class of pseudocontractive mappings with fixed points includes the class of demicontractive mappings.

Lemma 7 (see [19]). Let $\mathbb{U}$ be a real Hilbert space; let $\mathbb{C} \subset$ $\mathbb{W}$ be a closed convex set. Let $W: \mathbb{C} \rightarrow \mathbb{C}$ be a continuous pseudocontractive mapping. Then

(i) $\operatorname{Fix}(W)$ is a closed convex subset of $\mathbb{C}$;

(ii) $(I-W)$ is demiclosed at zero.

\section{Main Results}

Let $\mathbb{U}_{1}$ and $\mathbb{\boxplus}_{2}$ be two real Hilbert spaces and let $A: \mathbb{\mathbb { U } _ { 1 }} \rightarrow \mathbb{U}_{2}$ be a bounded linear operator with its adjoint $A^{*}$. This section is devoted to study problem (5), where $S: \mathbb{W}_{1} \rightarrow \mathbb{U}_{1}$ and

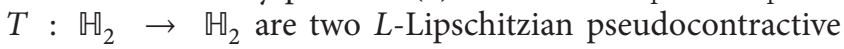
mappings. We denote the solution set of problem (5) by

$$
\Gamma=\left\{x^{\dagger} \in \operatorname{Fix}(S) ; A x^{\dagger} \in \operatorname{Fix}(T)\right\} .
$$

In the sequel, we assume $\Gamma \neq \emptyset$.

In order to solve problem (5), we present the following iterative algorithm. 
Algorithm 8. Let $\omega, \tau, \sigma, \varrho$, and $\varsigma$ be five constants. For $u \in \mathbb{H}_{1}$, arbitrarily, we define the following iterative manner:

$$
\begin{gathered}
\mathbb{C}_{1}=\mathbb{H}_{1}, \quad x_{1}=\operatorname{proj}_{\mathbb{C}_{1}}(u), \\
z_{n}=x_{n}+\omega A^{*}[(1-\tau) I+\tau T((1-\sigma) I+\sigma T)-I] A x_{n}, \\
y_{n}=(1-\varrho) z_{n}+\varrho S\left[(1-\varsigma) z_{n}+\varsigma S z_{n}\right], \\
\mathbb{C}_{n+1}=\left\{z \in \mathbb{C}_{n}:\left\|y_{n}-z\right\| \leq\left\|x_{n}-z\right\|\right\}, \\
x_{n+1}=\operatorname{proj}_{\mathbb{C}_{n+1}}(u), \quad n \in \mathbb{N} .
\end{gathered}
$$

Theorem 9. Assume that $\omega, \tau, \sigma, \varrho$, and $\varsigma$ satisfy the following assumptions: $0<\omega<1 /\|A\|^{2}, 0<\tau \leq \sigma<1 /\left(\sqrt{1+L^{2}}+1\right)$, and $0<\varrho<\varsigma<1 /\left(\sqrt{1+L^{2}}+1\right)$. Then, $\left\{x_{n}\right\},\left\{y_{n}\right\}$, and $\left\{z_{n}\right\}$ defined by (16) converge strongly to $\operatorname{proj}_{\Gamma}(u)$.

Proof. The outline of our proof details is as follows:

(i) $\Gamma \subset \mathbb{C}_{n}$, for all $n \in \mathbb{N}$;

(ii) $\mathbb{C}_{n}$ is closed and convex, for all $n \in \mathbb{N}$;

(iii) $x_{n} \rightarrow x^{*}$

(iv) $x^{*}=\operatorname{proj}_{\Gamma}(u)$.

Proof of (i). We show this by induction. (1) $\Gamma \subset \mathbb{C}_{1}=\mathbb{H}_{1}$ is obvious.

(2) Suppose that $\Gamma \subset \mathbb{C}_{k}$ for some $k \in \mathbb{N}$. For any $p \in \Gamma \subset$ $\mathbb{C}_{k}$, we have, from (14), that

$$
\begin{aligned}
\| S( & \left.(1-\varsigma) z_{k}+\varsigma S z_{k}\right)-p \|^{2} \\
= & \left\|S\left((1-\varsigma) z_{k}+\varsigma S z_{k}\right)-S p\right\|^{2} \\
\leq & \left\|(1-\varsigma) z_{k}+\varsigma S z_{k}-p\right\|^{2} \\
& +\left\|(1-\varsigma) z_{k}+\varsigma S z_{k}-S\left((1-\varsigma) z_{k}+\varsigma S z_{k}\right)\right\|^{2}, \\
& \left\|S z_{k}-p\right\|^{2} \leq\left\|z_{k}-p\right\|^{2}+\left\|z_{k}-S z_{k}\right\|^{2} .
\end{aligned}
$$

Observing that in any Hilbert space, we have

$$
\begin{array}{r}
\|t x+(1-t) y\|^{2}=t\|x\|^{2}+(1-t)\|y\|^{2}-t(1-t)\|x-y\|^{2} \\
\forall t \in[0,1]
\end{array}
$$

Set $v_{k}=(1-\varsigma) z_{k}+\varsigma S z_{k}$, for all $k \in \mathbb{N}$. By (17) and (18), we obtain

$$
\begin{aligned}
\left\|S v_{k}-p\right\|^{2} \leq & \left\|(1-\varsigma)\left(z_{k}-p\right)+\varsigma\left(S z_{k}-p\right)\right\|^{2} \\
& +\left\|(1-\varsigma) z_{k}+\varsigma S z_{k}-S v_{k}\right\|^{2}
\end{aligned}
$$

$$
\begin{aligned}
= & \left\|(1-\varsigma)\left(z_{k}-S v_{k}\right)+\varsigma\left(S z_{k}-S v_{k}\right)\right\|^{2} \\
& +\left\|(1-\varsigma)\left(z_{k}-p\right)+\varsigma\left(S z_{k}-p\right)\right\|^{2} \\
= & (1-\varsigma)\left\|z_{k}-S v_{k}\right\|^{2}+\varsigma\left\|S z_{k}-S v_{k}\right\|^{2} \\
& -\varsigma(1-\varsigma)\left\|z_{k}-S z_{k}\right\|^{2} \\
& +(1-\varsigma)\left\|z_{k}-p\right\|^{2}+\varsigma\left\|S z_{k}-p\right\|^{2} \\
& -\varsigma(1-\varsigma)\left\|z_{k}-S z_{k}\right\|^{2} \\
\leq & (1-\varsigma)\left\|z_{k}-p\right\|^{2}+\varsigma\left(\left\|z_{k}-p\right\|^{2}+\left\|z_{k}-S z_{k}\right\|^{2}\right) \\
& -2 \varsigma(1-\varsigma)\left\|z_{k}-S z_{k}\right\|^{2}+(1-\varsigma)\left\|z_{k}-S v_{k}\right\|^{2} \\
& +\varsigma\left\|S z_{k}-S v_{k}\right\|^{2} .
\end{aligned}
$$

Since $S$ is $L$-Lipschitzian and $z_{k}-v_{k}=\varsigma\left(z_{k}-S z_{k}\right)$, we have

$$
\begin{aligned}
\left\|S v_{k}-p\right\|^{2} \leq & (1-\varsigma)\left\|z_{k}-p\right\|^{2}+\varsigma\left(\left\|z_{k}-p\right\|^{2}+\left\|z_{k}-S z_{k}\right\|^{2}\right) \\
& -2 \varsigma(1-\varsigma)\left\|z_{k}-S z_{k}\right\|^{2}+(1-\varsigma)\left\|z_{k}-S v_{k}\right\|^{2} \\
& +\varsigma^{3} L^{2}\left\|z_{k}-S z_{k}\right\|^{2} \\
= & \left\|z_{k}-p\right\|^{2}+(1-\varsigma)\left\|z_{k}-S v_{k}\right\|^{2} \\
& -\varsigma\left(1-2 \varsigma-\varsigma^{2} L^{2}\right)\left\|z_{k}-S z_{k}\right\|^{2} .
\end{aligned}
$$

Since $\varsigma<1 /\left(\sqrt{1+L^{2}}+1\right)$, we deduce

$$
1-2 \varsigma-\varsigma^{2} L^{2}>0 \text {. }
$$

This together with (20) implies that

$$
\left\|S v_{k}-p\right\|^{2} \leq\left\|z_{k}-p\right\|^{2}+(1-\varsigma)\left\|z_{k}-S v_{k}\right\|^{2} .
$$

Hence,

$$
\begin{aligned}
\left\|y_{k}-p\right\|^{2}= & \left\|(1-\varrho) z_{k}+\varrho S v_{k}-p\right\|^{2} \\
= & \left\|(1-\varrho)\left(z_{k}-p\right)+\varrho\left(S v_{k}-p\right)\right\|^{2} \\
= & (1-\varrho)\left\|z_{k}-p\right\|^{2}+\varrho\left\|S v_{k}-p\right\|^{2} \\
& -\varrho(1-\varrho)\left\|S v_{k}-z_{k}\right\|^{2} \\
\leq & (1-\varrho)\left\|z_{k}-p\right\|^{2} \\
& +\varrho\left[\left\|z_{k}-p\right\|^{2}+(1-\varsigma)\left\|z_{k}-S v_{k}\right\|^{2}\right] \\
& -\varrho(1-\varrho)\left\|S v_{k}-z_{k}\right\|^{2} \\
= & \left\|z_{k}-p\right\|^{2}+(1-\varrho)(\varrho-\varsigma)\left\|S v_{k}-z_{k}\right\|^{2} .
\end{aligned}
$$

Noting that $\varrho \leq \varsigma$, we deduce

$$
\begin{aligned}
\left\|y_{k}-p\right\| & =\left\|(1-\varrho) z_{k}+\varrho S\left((1-\varsigma) z_{k}+\varsigma S z_{k}\right)-p\right\| \\
& \leq\left\|z_{k}-p\right\| .
\end{aligned}
$$


Similarly, we also have

$\left\|[(1-\tau) I+\tau T((1-\sigma) I+\sigma T)] A x_{k}-A p\right\| \leq\left\|A x_{k}-A p\right\|$

In Hilbert spaces, there holds

$$
2\left\langle z^{\dagger}, z\right\rangle=\left\|z^{\dagger}+z\right\|^{2}-\left\|z^{\dagger}\right\|^{2}-\|z\|^{2}
$$

With the help of (25) and (26), we get

$$
\begin{aligned}
& \left\|z_{k}-p\right\|^{2} \\
& =\| x_{k}-p+\omega A^{*}[(1-\tau) I+\tau T((1-\sigma) I+\sigma T)-I] \\
& \times A x_{k} \|^{2} \\
& =2 \omega\left\langle A^{*}[(1-\tau) I+\tau T((1-\sigma) I+\sigma T)-I]\right. \\
& \left.\times A x_{k}, x_{k}-p\right\rangle \\
& +\omega^{2}\left\|A^{*}[(1-\tau) I+\tau T((1-\sigma) I+\sigma T)-I] A x_{k}\right\|^{2} \\
& +\left\|x_{k}-p\right\|^{2} \\
& =2 \omega\left\langle[(1-\tau) I+\tau T((1-\sigma) I+\sigma T)-I] A x_{k},\right. \\
& \left.A x_{k}-A p\right\rangle \\
& +\omega^{2}\|A\|^{2}\left\|[(1-\tau) I+\tau T((1-\sigma) I+\sigma T)-I] A x_{k}\right\|^{2} \\
& +\left\|x_{k}-p\right\|^{2} \\
& =\left\|x_{k}-p\right\|^{2} \\
& +\omega\left[\left\|[(1-\tau) I+\tau T((1-\sigma) I+\sigma T)] A x_{k}-A p\right\|^{2}\right. \\
& -\left\|[(1-\tau) I+\tau T((1-\sigma) I+\sigma T)-I] A x_{k}\right\|^{2} \\
& \left.-\left\|A x_{k}-A p\right\|^{2}\right] \\
& +\omega^{2}\|A\|^{2}\left\|[(1-\tau) I+\tau T((1-\sigma) I+\sigma T)-I] A x_{k}\right\|^{2} \\
& \leq \omega\left(\omega\|A\|^{2}-1\right) \|[(1-\tau) I+\tau T((1-\sigma) I+\sigma T)-I] \\
& \times A x_{k} \|^{2} \\
& +\left\|x_{k}-p\right\|^{2} \leq\left\|x_{k}-p\right\|^{2}
\end{aligned}
$$

By (24) and (27), we have

$$
\left\|y_{k}-p\right\| \leq\left\|z_{k}-p\right\| \leq\left\|x_{k}-p\right\|
$$

This shows that $p \in \mathbb{C}_{k+1}$. Thus, we get $\Gamma \subset \mathbb{C}_{n}$, for all $n \in \mathbb{N}$.
Proof of (ii). It is easy to verify that $\mathbb{C}_{n}$ is closed, for all $n \in \mathbb{N}$. Next, we only need to verify that $\mathbb{C}_{n}$ is convex, for all $n \in \mathbb{N}$. In fact, let $z^{\dagger}, z^{\ddagger} \in \mathbb{C}_{n+1}$; for each $\zeta \in(0,1)$, we have

$$
\begin{aligned}
\left\|y_{n}-\left(\zeta z^{\dagger}+(1-\zeta) z^{\ddagger}\right)\right\|^{2}= & \left\|\zeta\left(y_{n}-z^{\dagger}\right)+(1-\zeta)\left(y_{n}-z^{\ddagger}\right)\right\|^{2} \\
= & \zeta\left\|y_{n}-z^{\dagger}\right\|^{2}+(1-\zeta)\left\|y_{n}-z^{\ddagger}\right\|^{2} \\
& -\zeta(1-\zeta)\left\|z^{\dagger}-z^{\ddagger}\right\|^{2} \\
\leq & \zeta\left\|z_{n}-z^{\dagger}\right\|^{2}+(1-\zeta)\left\|z_{n}-z^{\ddagger}\right\|^{2} \\
& -\zeta(1-\zeta)\left\|z^{\dagger}-z^{\ddagger}\right\|^{2} \\
= & \left\|z_{n}-\left(\zeta z^{\dagger}+(1-\zeta) z^{\ddagger}\right)\right\|^{2} ;
\end{aligned}
$$

namely,

$$
\left\|y_{n}-\left(\zeta z^{\dagger}+(1-\zeta) z^{\ddagger}\right)\right\| \leq\left\|z_{n}-\left(\zeta z^{\dagger}+(1-\zeta) z^{\ddagger}\right)\right\| ;
$$

this shows $\zeta z^{\dagger}+(1-\zeta) z^{\ddagger} \in \mathbb{C}_{n+1}$ and $\mathbb{C}_{n+1}$ is a convex set, for all $n \in \mathbb{N}$.

Proof of (iii). Since $\Gamma \subset \mathbb{C}_{n+1} \subset \mathbb{C}_{n}$ and $x_{n+1}=P_{\mathbb{C}_{n+1}}(u) \subset \mathbb{C}_{n}$, we obtain

$$
\left\|x_{n+1}-u\right\| \leq\|p-u\| \quad \forall n \in \mathbb{N}, p \in \Gamma .
$$

It follows that $\left\{x_{n}\right\}$ is bounded.

It is known that the metric projection $\operatorname{proj}_{\mathbb{C}}$ can be characterized by

$$
\left\langle x^{\dagger}-\operatorname{proj}_{\mathbb{C}}\left(x^{\dagger}\right), \operatorname{proj}_{\mathbb{C}}\left(x^{\dagger}\right)-x\right\rangle \geq 0, \quad \forall x \in \mathbb{C} ;
$$

equivalently,

$$
\left\|x-\operatorname{proj}_{\mathbb{C}}\left(x^{\dagger}\right)\right\|^{2}+\left\|x^{\dagger}-\operatorname{proj}_{\mathbb{C}}\left(x^{\dagger}\right)\right\|^{2} \leq\left\|x-x^{\dagger}\right\|^{2} .
$$

With the help of (33), we have

$$
\begin{aligned}
\left\|x_{n+1}-x_{n}\right\|^{2}+\left\|u-x_{n}\right\|^{2} & =\left\|x_{n+1}-P_{\mathbb{C}_{n}}(u)\right\|^{2}+\left\|u-P_{\mathbb{C}_{n}}(u)\right\|^{2} \\
& \leq\left\|x_{n+1}-u\right\|^{2}
\end{aligned}
$$

which implies that

$$
0 \leq\left\|x_{n+1}-x_{n}\right\|^{2} \leq\left\|x_{n+1}-u\right\|^{2}-\left\|u-x_{n}\right\|^{2} .
$$

It follows that

$$
\left\|x_{n}-u\right\| \leq\left\|x_{n+1}-u\right\|
$$

Since $\left\{\left\|x_{n}-u\right\|\right\}$ is bounded, we get

$$
\lim _{n \rightarrow \infty}\left\|x_{n}-u\right\| \text { exists. }
$$


This together with (35) implies that

$$
\lim _{n \rightarrow \infty}\left\|x_{n+1}-x_{n}\right\|=0 \text {. }
$$

The fact that $x_{n+1}=P_{\mathbb{C}_{n+1}}(u) \in \mathbb{C}_{n+1}$ gives

$$
\left\|y_{n}-x_{n+1}\right\| \leq\left\|x_{n}-x_{n+1}\right\| \longrightarrow 0 .
$$

By (38) and (39), we derive

$$
\lim _{n \rightarrow \infty}\left\|y_{n}-x_{n}\right\|=0 \text {. }
$$

Next we show that $\left\{x_{n}\right\}$ is a Cauchy sequence. As a matter of fact, for any $m, n \in \mathbb{N}$ with $m>n$, we have

$$
\begin{aligned}
\left\|x_{m}-x_{n}\right\|^{2}+\left\|x_{n}-u\right\|^{2} & =\left\|x_{m}-P_{\mathbb{C}_{n}}(u)\right\|^{2}+\left\|P_{\mathbb{C}_{n}}(u)-u\right\|^{2} \\
& \leq\left\|x_{m}-u\right\|^{2} .
\end{aligned}
$$

It follows that

$$
\left\|x_{m}-x_{n}\right\|^{2} \leq\left\|x_{m}-u\right\|^{2}-\left\|x_{n}-u\right\|^{2} .
$$

Note that $\lim _{n \rightarrow \infty}\left(\left\|x_{m}-u\right\|^{2}-\left\|x_{n}-u\right\|^{2}\right)=0$. Therefore, $\lim _{n \rightarrow \infty}\left\|x_{m}-x_{n}\right\|=0$. So, $\left\{x_{n}\right\}$ is a Cauchy sequence and hence $x_{n} \rightarrow x^{*}$.

Proof of (iv). From (24), (27), and (40), we have

$$
\begin{aligned}
- & \omega\left(\omega\|A\|^{2}-1\right)\left\|[(1-\tau) I+\tau T((1-\sigma) I+\sigma T)-I] A x_{n}\right\|^{2} \\
& \leq\left\|x_{n}-p\right\|^{2}-\left\|y_{n}-p\right\|^{2} \\
& \leq\left\|x_{n}-y_{n}\right\|\left(\left\|x_{n}-p\right\|+\left\|y_{n}-p\right\|\right) \\
& \longrightarrow 0 .
\end{aligned}
$$

Hence,

$$
\lim _{n \rightarrow \infty}\left\|[(1-\tau) I+\tau T((1-\sigma) I+\sigma T)-I] A x_{n}\right\|=0 .
$$

It follows that

$$
\lim _{n \rightarrow \infty}\left\|T((1-\sigma) I+\sigma T) A x_{n}-A x_{n}\right\|=0 .
$$

Next, we firstly show that

$$
\operatorname{Fix}(T)=\operatorname{Fix}(T((1-\sigma) I+\sigma T)) .
$$

As a matter of fact, $\operatorname{Fix}(T) \subset \operatorname{Fix}(T((1-\sigma) I+\sigma T))$ is obvious. We only need to show that $\operatorname{Fix}(T((1-\sigma) I+\sigma T)) \subset \operatorname{Fix}(T)$.

Take any $y^{\dagger} \in \operatorname{Fix}(T((1-\sigma) I+\sigma T))$. We have $T((1-\sigma) I+$ $\sigma T) y^{\dagger}=y^{\dagger}$. Set $R=(1-\sigma) I+\sigma T$. We have $T R y^{\dagger}=y^{\dagger}$. Write $R y^{\dagger}=y^{*}$. Then, $T y^{*}=y^{\dagger}$. Next, we show $y^{\dagger}=y^{*}$. In fact,

$$
\begin{aligned}
\left\|y^{\dagger}-y^{*}\right\| & =\left\|T y^{*}-R y^{\dagger}\right\| \\
& =\left\|T y^{*}-(1-\sigma) y^{\dagger}-\sigma T y^{\dagger}\right\| \\
& =\sigma\left\|T y^{*}-T y^{\dagger}\right\| \\
& \leq \sigma L\left\|y^{*}-y^{\dagger}\right\| .
\end{aligned}
$$

Since $\sigma<1 /\left(\sqrt{1+L^{2}}+1\right)<1 / L$, we deduce $y^{*}=y^{\dagger} \epsilon$ $\operatorname{Fix}(R)=\operatorname{Fix}(T)$. Thus, $y^{\dagger} \in \operatorname{Fix}(T)$. Hence, $\operatorname{Fix}(T((1-\sigma) I+$ $\sigma T)) \subset \operatorname{Fix}(T)$. Therefore, $\operatorname{Fix}(T((1-\sigma) I+\sigma T))=\operatorname{Fix}(T)$.

With (46) in hand, we prove that $T((1-\sigma) I+\sigma T)-I$ is demiclosed at 0 . Let the sequence $\left\{u_{n}\right\} \subset \mathbb{M}_{2}$ satisfying $u_{n} \rightarrow$ $y^{\dagger}$ and $u_{n}-T((1-\sigma) I+\sigma T) u_{n} \rightarrow 0$. Now, we show that $y^{\dagger} \in \operatorname{Fix}(T((1-\sigma) I+\sigma T))$.

Since $T$ is $L$-Lipschitzian, we have

$$
\begin{aligned}
\left\|u_{n}-T u_{n}\right\| \leq & \left\|u_{n}-T((1-\sigma) I+\sigma T) u_{n}\right\| \\
& +\left\|T((1-\sigma) I+\sigma T) u_{n}-T u_{n}\right\| \\
\leq & \left\|u_{n}-T((1-\sigma) I+\sigma T) u_{n}\right\|+\sigma L\left\|u_{n}-T u_{n}\right\| .
\end{aligned}
$$

It follows that

$$
\left\|u_{n}-T u_{n}\right\| \leq \frac{1}{1-\sigma L}\left\|u_{n}-T((1-\sigma) I+\sigma T) u_{n}\right\|
$$

Hence,

$$
\lim _{n \rightarrow \infty}\left\|u_{n}-T u_{n}\right\|=0
$$

Since $T-I$ is demiclosed at 0 by Lemma 7, we immediately deduce $y^{\dagger} \in \operatorname{Fix}(T)=\operatorname{Fix}(T((1-\sigma) I+\sigma T))$. Therefore, $T((1-$ $\sigma) I+\sigma T)-I$ is demiclosed at 0 . Since $A$ is a bounded linear operator, we get $\left\|A x_{n}-A x^{*}\right\| \rightarrow 0$. From (45), we deduce $A x^{*} \in \operatorname{Fix}(T((1-\sigma) I+\sigma T))=\operatorname{Fix}(T)$.

By (16), (40), and (44), we deduce

$$
\lim _{n \rightarrow \infty}\left\|z_{n}-y_{n}\right\|=0
$$

So,

$$
\lim _{n \rightarrow \infty}\left\|S\left[(1-\varsigma) z_{n}+\varsigma S z_{n}\right]-z_{n}\right\|=\lim _{n \rightarrow \infty} \frac{1}{\varrho}\left\|y_{n}-z_{n}\right\|=0
$$

Similarly, we can show that $x^{*} \in \operatorname{Fix}(S)$. To this end, we have proven that $x^{*} \in \operatorname{Fix}(S)$ and $A x^{*} \in \operatorname{Fix}(T)$. Therefore, $x^{*} \in \Gamma$. This completes the proof.

\section{Conflict of Interests}

The authors declare that there is no conflict of interests regarding the publication of this paper.

\section{Acknowledgments}

The third author was supported by the fund of the Research Promotion Program, Gyeongsang National University, 2013 (RPP-2013-023). The fourth author was supported in part by the NNSF of China (61362033) and NZ13087. 


\section{References}

[1] H. Stark, Ed., Image Recovery Theory and Applications, Academic Press, Orlando, Fla, USA, 1987.

[2] P. L. Combettes, "The convex feasibility problem in image recovery," in Advances in Imaging and Electron Physics, P. Hawkes, Ed., vol. 95, pp. 155-270, Academic Press, New York, NY, USA, 1996.

[3] H. H. Bauschke and J. M. Borwein, "On projection algorithms for solving convex feasibility problems," SIAM Review, vol. 38, no. 3, pp. 367-426, 1996.

[4] Y. Censor and T. Elfving, "A multiprojection algorithm using Bregman projections in a product space," Numerical Algorithms, vol. 8, no. 2-4, pp. 221-239, 1994.

[5] C. Byrne, "Iterative oblique projection onto convex sets and the split feasibility problem," Inverse Problems. An International Journal on the Theory and Practice of Inverse Problems, Inverse Methods and Computerized Inversion of Data, vol. 18, no. 2, pp. 441-453, 2002.

[6] H.-K. Xu, "Iterative methods for the split feasibility problem in infinite-dimensional Hilbert spaces," Inverse Problems. An International Journal on the Theory and Practice of Inverse Problems, Inverse Methods and Computerized Inversion of Data, vol. 26, no. 10, 17 pages, 2010.

[7] J. Zhao and Q. Yang, "Several solution methods for the split feasibility problem," Inverse Problems. An International Journal on the Theory and Practice of Inverse Problems, Inverse Methods and Computerized Inversion of Data, vol. 21, no. 5, pp. 1791-1799, 2005.

[8] Y. Dang and Y. Gao, "The strong convergence of a KM-CQlike algorithm for a split feasibility problem," Inverse Problems. An International Journal on the Theory and Practice of Inverse Problems, Inverse Methods and Computerized Inversion of Data, vol. 27, no. 1, 9 pages, 2011.

[9] F. Wang and H.-K. Xu, "Approximating curve and strong convergence of the CQ algorithm for the split feasibility problem," Journal of Inequalities and Applications, vol. 2010, Article ID 102085, 13 pages, 2010.

[10] Y. Yao, W. Jigang, and Y.-C. Liou, "Regularized methods for the split feasibility problem," Abstract and Applied Analysis, vol. 2012, Article ID 140679, 15 pages, 2012.

[11] L.-C. Ceng, Q. H. Ansari, and J.-C. Yao, "Relaxed extragradient methods for finding minimum-norm solutions of the split feasibility problem," Nonlinear Analysis: Theory, Methods \& Applications, vol. 75, no. 4, pp. 2116-2125, 2012.

[12] Y. Yao, T. H. Kim, S. Chebbi, and H.-K. Xu, "A modified extragradient method for the split feasibility and fixed point problems," Journal of Nonlinear and Convex Analysis, vol. 13, no. 3, pp. 383-396, 2012.

[13] Y. Yao, M. Postolache, and Y.-C. Liou, "Strong convergence of a self-adaptive method for the split feasibility problem," Fixed Point Theory and Applications, vol. 2013, 13 pages, 2013.

[14] Y. Censor and A. Segal, "The split common fixed point problem for directed operators," Journal of Convex Analysis, vol. 16, no. 2, pp. 587-600, 2009.

[15] A. Moudafi, "The split common fixed-point problem for demicontractive mappings," Inverse Problems. An International Journal on the Theory and Practice of Inverse Problems, Inverse Methods and Computerized Inversion of Data, vol. 26, no. 5, 6 pages, 2010.
[16] Z. H. He and W. S. Du, "Nonlinear algorithms approach to split common solution problems," Fixed Point Theory and Applications, vol. 130, 14 pages, 2012.

[17] H. Cui, M. Su, and F. Wang, "Damped projection method for split common fixed point problems," Journal of Inequalities and Applications, vol. 2013, 10 pages, 2013.

[18] S. Maruster and C. Popirlan, "On the Mann-type iteration and the convex feasibility problem," Journal of Computational and Applied Mathematics, vol. 212, no. 2, pp. 390-396, 2008.

[19] H. Zhou, "Strong convergence of an explicit iterative algorithm for continuous pseudo-contractions in Banach spaces," Nonlinear Analysis: Theory, Methods \& Applications, vol. 70, no. 11, pp. 4039-4046, 2009. 


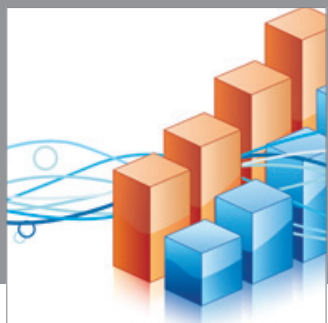

Advances in

Operations Research

mansans

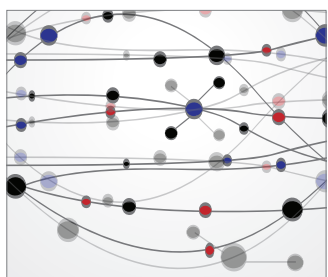

The Scientific World Journal
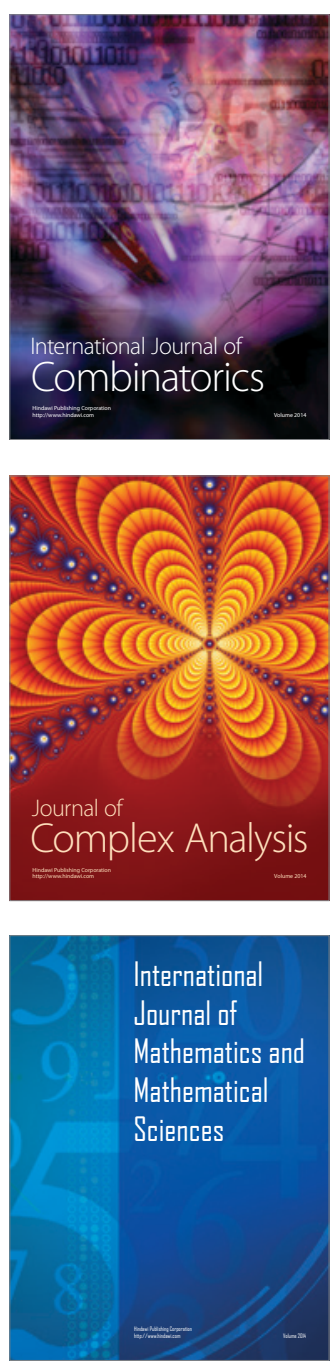
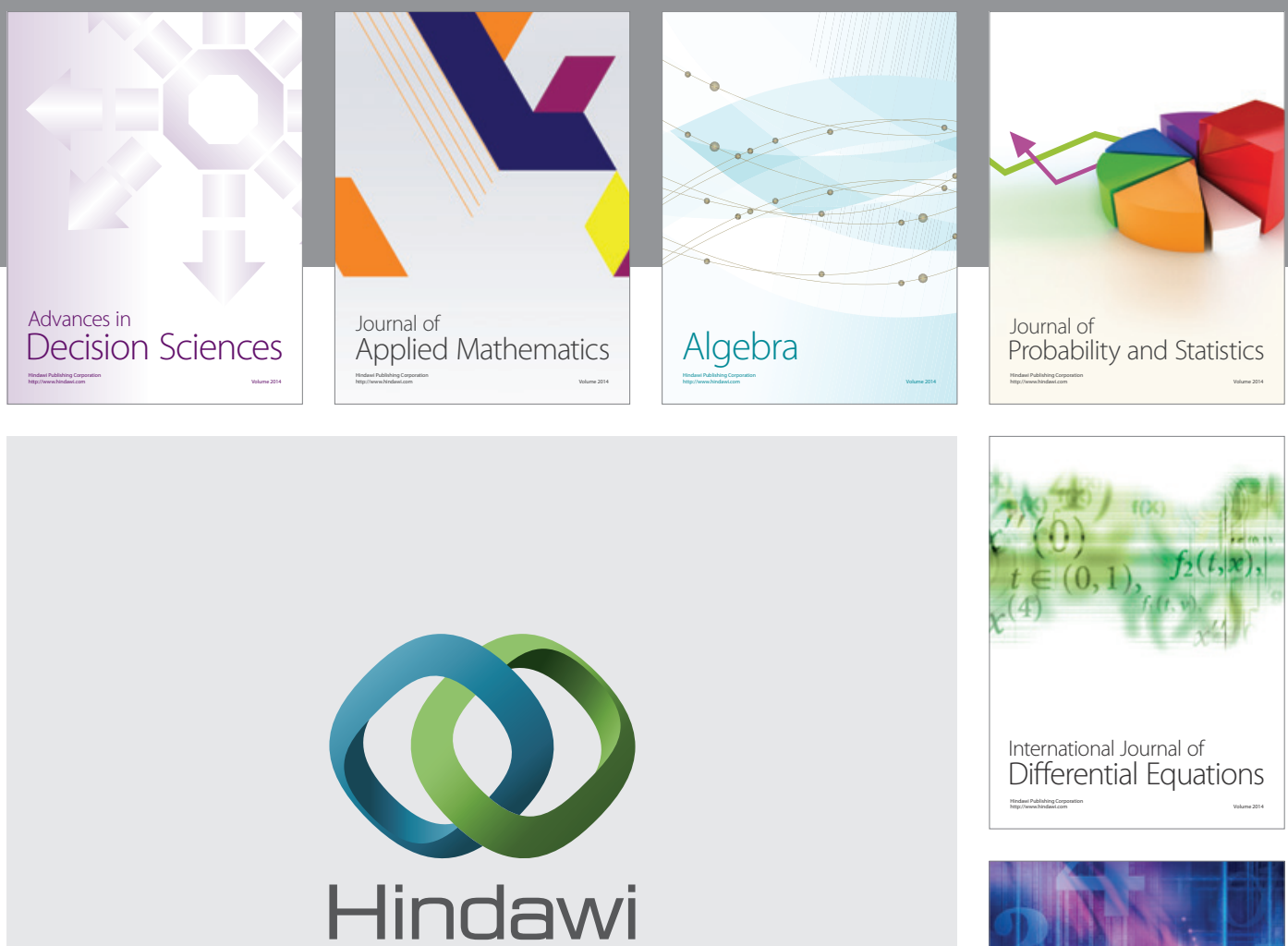

Submit your manuscripts at http://www.hindawi.com
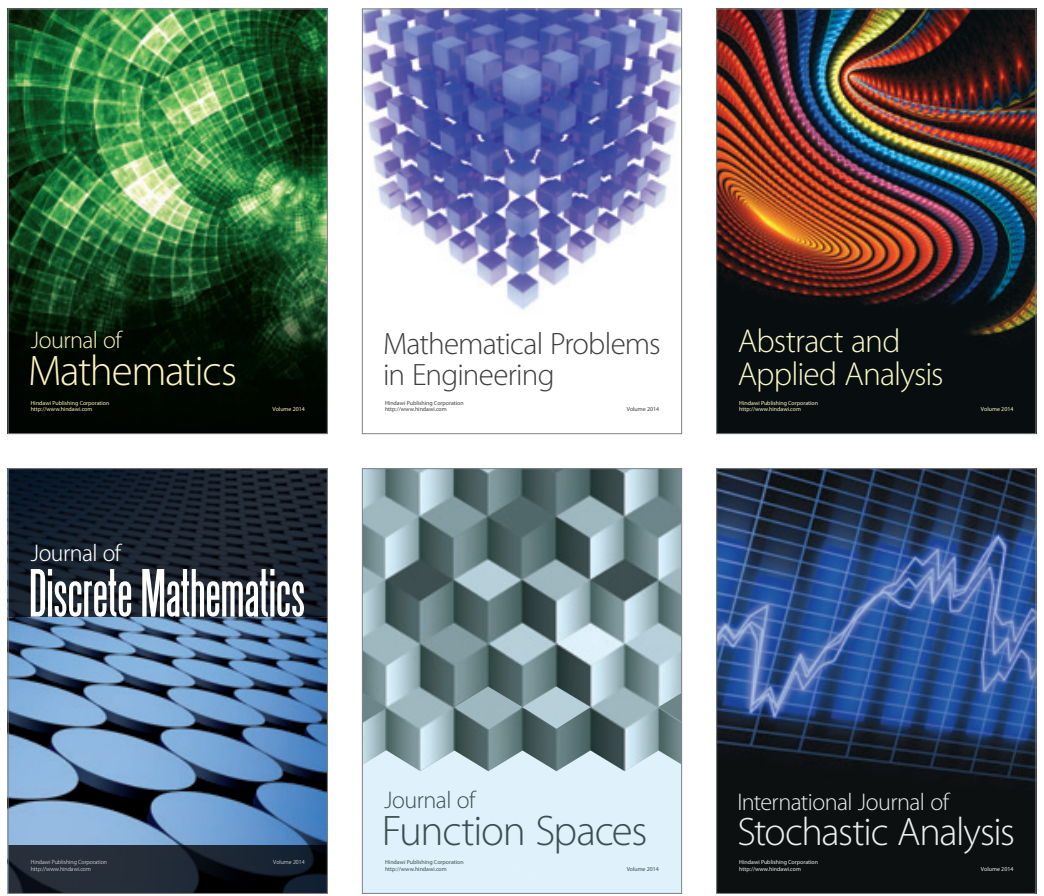

Journal of

Function Spaces

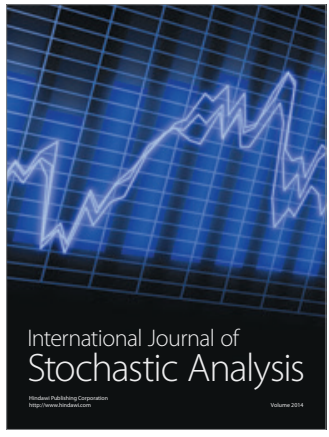

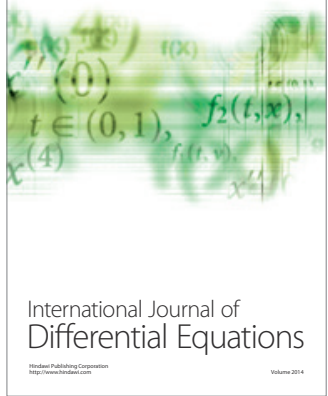
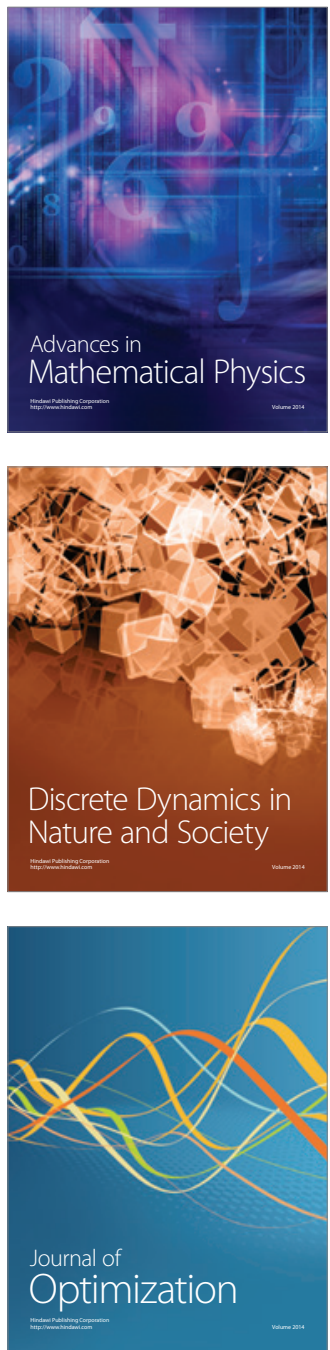